\title{
Dexamethasone nanomedicines for COVID-19
}

\author{
Nano-formulating dexamethasone, and administering it via intravenous injection or inhalation, may help to improve \\ anti-COVID-19 treatment efficacy by targeting the potent corticosteroid drug to hyper-activated immune cells, \\ by potentiating its anti-oedema activity and by exploiting its anti-fibrotic effects.
}

\section{Twan Lammers, Alexandros Marios Sofias, Roy van der Meel, Raymond Schiffelers, Gert Storm, Frank Tacke, Steffen Koschmieder, Tim H. Brümmendorf, Fabian Kiessling and Josbert M. Metselaar}

$\mathrm{D}$ examethasone is the first drug to show life-saving efficacy in patients infected with COVID-19. In the world's largest randomized controlled trial (RCT) of COVID-19 treatments, the so-called RECOVERY trial ${ }^{1}$, six interventions are being evaluated. Besides the potent anti-inflammatory corticosteroid dexamethasone, these include the anti-malaria medication hydroxychloroquine, the antibiotic azithromycin, the anti-HIV drug cocktail lopinavir-ritonavir, the anti-inflammatory antibody tocilizumab, and convalescent plasma from cured patients. The first and the third statement published by the chief investigators of the RECOVERY trial reported no clinical benefit for hydroxychloroquine and lopinavir-ritonavir in hospitalized patients ${ }^{1}$. The second statement announced that dexamethasone (6 $\mathrm{mg} \mathrm{day}^{-1}$; administered orally or intravenously for 10 days) reduces the numbers of COVID-19-related deaths by $35 \%$ in patients on the intensive care unit (ICU) who require mechanical ventilation ${ }^{1,2}$. In non-ventilated patients on oxygen therapy, the mortality rate was reduced by $20 \%$. Moreover, dexamethasone treatment resulted in a shorter hospitalization time (12 days for the dexamethasone group versus 13 days for standard-of-care), and in a higher probability of hospital discharge within the 28 days of the clinical trial $(65 \% \text { versus } 61 \%)^{2}$. These findings are in line with recently published findings on dexamethasone efficacy in acute respiratory distress syndrome ${ }^{3}$, and they are expected to have massive global impact. Not only because dexamethasone is the first and thus far only drug to significantly improve survival in a RCT in COVID-19 patients, but also because it is a very well-known and extensively used drug, which is widely available and very cheap ${ }^{4}$.

While the main focus of initial RCT against COVID-19 has been on antiviral drugs, such as remdesivir, it has become clear that a significant number of severe cases and fatalities are due to an overreaction of the immune system, leading to hyperinflammation and to macrophage activation syndrome (MAS). It is important to note in this regard that the recent results from the RECOVERY trial indicate that at 1 week post COVID-19 infection, the health status of patients is primarily debilitated by immunopathological phenomena, rather than by viral replication ${ }^{2}$. Hyperinflammation and MAS result in an overproduction of proinflammatory cytokines, such as IL-1 $\beta$, IL- 6 and TNF- $\alpha$ (that is, cytokine storm), as well as in coagulation abnormalities, which contribute to organ failure and fatalities ${ }^{5,6}$. Recognizing the importance of proinflammatory cytokines in COVID-19 severity and mortality, highly specific anti-cytokine biologics have been proposed as potential treatments for critically ill patients, including for example the anti-IL-6 antibody tocilizumab, which is included in the RECOVERY trial. While we are all eagerly awaiting further announcements from this and other ongoing RCT, it is striking that a well-known and widely available broad-spectrum cytokine suppressor drug, which is at least a hundred times cheaper than specific anti-cytokine antibodies, is the first to show live-saving efficacy against COVID-19. Sure, dexamethasone's mechanisms of action go way beyond suppressing cytokines. But in the current indication and situation, in which we cannot yet causally link disease progression and patient mortality to specific molecular features, this broad mechanism of action may actually be beneficial. Especially if we would be able to improve the delivery of dexamethasone to target cells and tissues that play a key role in the acute and progressive phase of COVID-19.

We here propose to nano-formulate dexamethasone to improve the management of COVID-19 complications. At the preclinical level, several different diseases have already been successfully treated with dexamethasone nanomedicines, including, for example, rheumatoid arthritis, inflammatory bowel disease, multiple sclerosis, liver fibrosis, wound healing and cancer ${ }^{7-12}$. In the case of cancer, liposomal dexamethasone has shown promising efficacy in syngeneic and xenograft mouse models, particularly also in multiple myeloma ${ }^{13}$, a disease in which dexamethasone has long been a cornerstone drug as part of both induction and maintenance therapy. At the University Medical Center at RWTH Aachen, a first-in-man clinical trial of PEGylated liposomal dexamethasone has been initiated in 2017 in patients with progressive multiple myeloma ${ }^{14}$. The results obtained thus far show good tolerability at doses up until $40 \mathrm{mg}$ (dexamethasone-equivalent), as well as initial signs of efficacy.

The proposition that dexamethasone nanomedicines are useful for the treatment of COVID-19 is based on the widely recognized notion that nanoparticles potently accumulate in macrophages, upon intravenous administration as well as upon inhalation (Fig. 1). In this context, it is worth mentioning the liposomal amikacin product Arikayce, which was approved by the US Food and Drug Administration in 2019 for treating Mycobacterium avium complex lung disease. As a nanomedicine formulation, Arikayce efficiently targets the pulmonary macrophages where the bacterial pathogen resides and it has been shown to thereby improve disease treatment as compared to free amikacin ${ }^{15}$. Along the same line of thinking, pulmonary delivery of dexamethasone liposomes may outperform free dexamethasone when targeting alveolar macrophages as a strategy to intervene in the (sub)acute phase of COVID-19. Intravenous administration, on the other hand, provides the possibility of using liposomes and other nanomedicine formulations to target dexamethasone to myeloid and lymphoid tissues that are enriched with phagocytes, such as the spleen and bone marrow. It furthermore enables efficient and relatively selective delivery of the potent corticosteroid drug to sites of inflammation where the vasculature is leaky and where large numbers of 

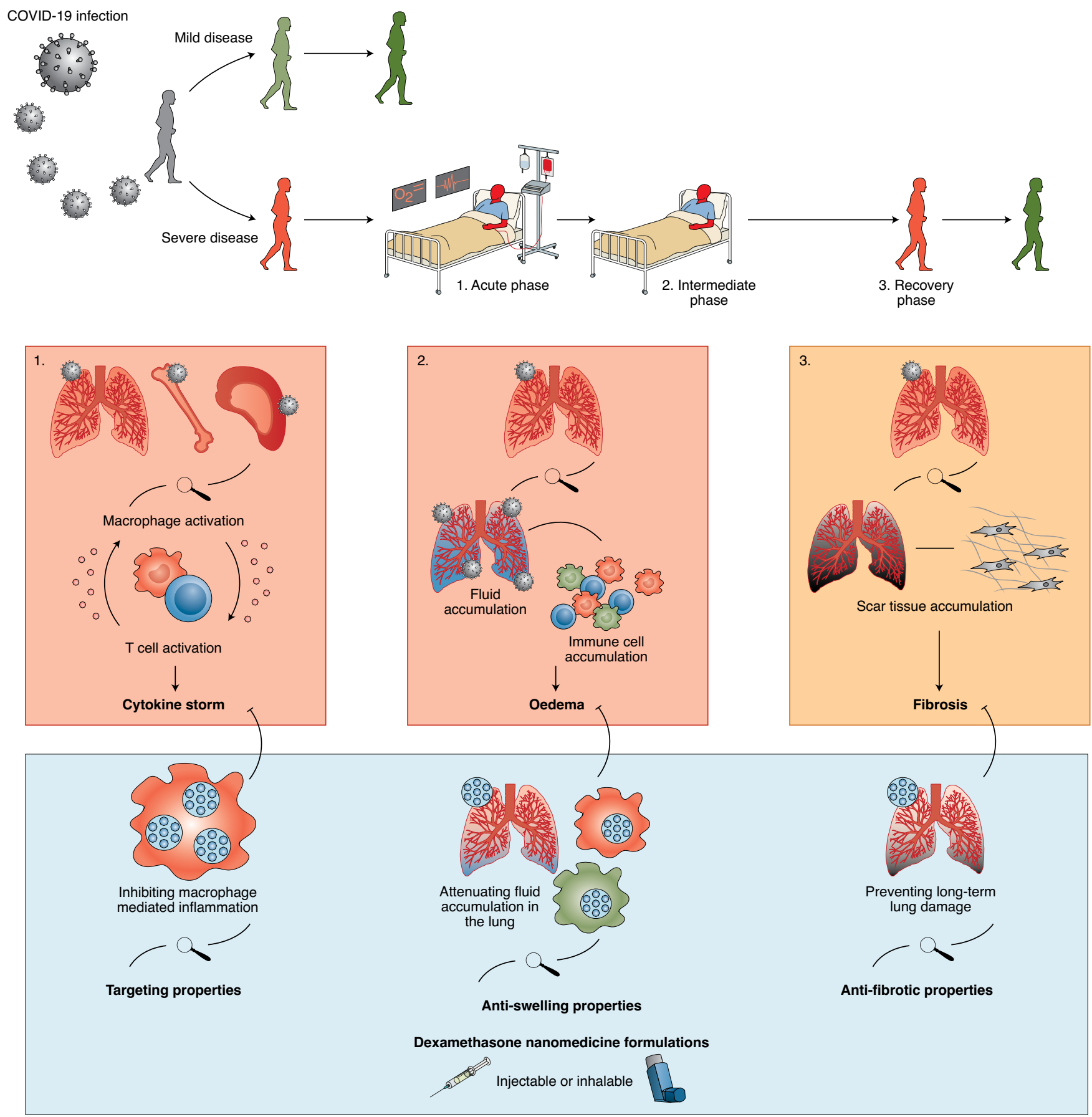

Fig. 1 | Dexamethasone nanomedicines for COVID-19. COVID-19 infection causes several acute and longer-lasting life-threatening symptoms, including cytokine storm, oedema formation and fibrosis development. Dexamethasone nanomedicines may help to better manage the severity of these disease manifestations, promoting better control of life-threatening symptoms at the acute and intermediate phase, and more rapid and more complete recovery in the post-ICU phase.

phagocytes have infiltrated, attenuating the production of proinflammatory cytokines, of matrix degrading enzymes and of other signalling molecules that contribute to oedema formation and progressive tissue damage in COVID-19. It is crucial in this regard to impart long-circulating behaviour (for example, via PEGylation) upon the intravenously injected nanomedicines, as this promotes accumulation in inflammatory macrophages infiltrated at the pathological site, while avoiding rapid capture by the liver- and spleen-resident macrophage populations that are responsible for clearing nanomedicine formulations from the blood stream ${ }^{16}$.
Dexamethasone nanomedicines are nowhere near a vaccine in terms of global impact and control of COVID-19 disease burden. In a number of cases, however, dexamethasone nanomedicines may help in the day-to-day management of the disease:

(1) As alluded to above, nanomedicine formulations can help to target the potent 
corticosteroid drug to inflammation-initiating and -propagating phagocytic cells in the lung, in the blood, and in myeloid and lymphoid tissues. This assists in better controlling MAS and cytokine storm, which have been implicated in COVID-19-related fatalities ${ }^{5}$. As a consequence, critically ill patients on ventilation or on oxygen therapy are expected to recover faster and more efficiently than upon treatment with the free drug.

(2) Dexamethasone is a highly active anti-oedema agent. Its potent anti-swelling properties contribute to its mechanism of action in multiple different diseases, including in high-grade inflammatory disorders and in glioblastoma, and this assumingly also contributes to its activity in COVID-19. Nanoformulating dexamethasone could further potentiate this effect, by increasing drug availability and drug activity over time in hyperactivated immune cell populations in the inflamed parts of the lung. Dexamethasone nanomedicine formulations may furthermore help to sustain anti-inflammatory and anti-oedema drug activity in the days and weeks after patients have been released from the hospital.

(3) Dexamethasone is a highly potent anti-fibrotic agent. Multiple preclinical studies, in various different disease models, have shown that the anti-fibrotic effects of dexamethasone can be potentiated by reformulating it as a nanomedicine formulation $^{7-12}$. In this context, dexamethasone nanomedicines have been shown to be particularly useful for preventing fibrosis. Since pulmonary fibrosis has recently emerged as a key complication in the long-term follow-up management of COVID-19 (especially in patients that have been ventilated for prolonged periods of time $)^{17}$, inhaled or intravenously injected dexamethasone nanomedicines could meet an urgent medical need also at this level of COVID-19 management.

When realistically reflecting upon the potential of dexamethasone nanomedicines for the treatment of COVID-19, money and time are critical issues to consider. The fact that dexamethasone is an already widely available and very cheap drug with - now proven - life-saving capability in COVID-19 substantially raises the bar for any new nanomedicine product based on dexamethasone. A dexamethasone nanomedicine product obviously entails a higher level of complexity in terms of composition and manufacturing, and it would first have to be clinically tested and registered before it becomes available on the market, where it would have to fetch at least US $\$ 100$ per treatment to make it economically viable. We believe that the key undertaking here is to carefully design the clinical studies with the nanomedicine product so as to unambiguously prove what the actual added value is. If targeted delivery of dexamethasone in COVID-19 patients using a nanomedicine formulation leads to better outcomes, for instance in terms of a reduction of the number of days that patients need mechanical ventilation and/or require costly ICU hospitalization ${ }^{18,19}$, then this is already a huge gain that can easily offset against the higher level of complexity and cost of the nanodrug. And if in these clinical studies, dexamethasone nanomedicines would also turn out to be able to outperform the free drug in terms of improving the survival of critically ill patients, then that would be another major leap forward in the worldwide battle against COVID-19.

Twan Lammers (D) 1,2,3凶,

Alexandros Marios Sofias (D)1,

Roy van der $\mathrm{Meel}^{4}$,

Raymond Schiffelers ${ }^{2,5}$, Gert Storm ${ }^{2,3,6}$, Frank Tacke ${ }^{7}$, Steffen Koschmieder (iD), Tim H. Brümmendorf ${ }^{8}$, Fabian Kiessling (D)1 and Josbert M. Metselaar ${ }^{1 \times}$

${ }^{I}$ Institute for Experimental Molecular Imaging, Faculty of Medicine, RWTH Aachen University, Aachen, Germany. ${ }^{2}$ Department of Pharmaceutics, Utrecht University, Utrecht, The Netherlands. ${ }^{3}$ Department of Targeted Therapeutics, University of Twente, Enschede, The Netherlands. ${ }^{4}$ Department of
Biomedical Engineering and Institute for Complex Molecular Systems, Eindhoven University of Technology, Eindhoven, The Netherlands. ${ }^{5}$ Laboratory of Clinical Chemistry and Hematology, University Medical Center Utrecht, Utrecht, The Netherlands. ${ }^{6}$ Department of Surgery, Yong Loo Lin School of Medicine, National University of Singapore, Kent Ridge, Singapore. ${ }^{7}$ Department of Hepatology and Gastroenterology, Charité University Medicine, Berlin, Germany. ${ }^{8}$ Department of Medicine IV (Hematology, Oncology, Hemostaseology, and Stem Cell Transplantation), RWTH Aachen

University, Aachen, Germany.

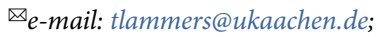
jmetselaar@ukaachen.de

Published online: 3 August 2020 https://doi.org/10.1038/s41565-020-0752-z

\section{References}

1. Results. RECOVERY Trial https://www.recoverytrial.net/results (2020)

2. Horby, P. et al. N. Engl. J. Med. https://doi.org/10.1056/ NEJMoa2021436 (2020)

3. Villar, J. et al. Lancet. Respir. Med. 8, 267-276 (2020).

4. Ledford, H. Nature https://doi.org/10.1038/d41586-020-01824-5 (2020).

5. Merad, M. \& Martin, J. C. Nat. Rev. Immunol. 20, 355-362 (2020).

6. Tay, M. Z., Poh, C. M., Rénia, L., MacAry, P. A. \& Ng, L. F. P. T. Nat. Rev. Immunol. 20, 363-374 (2020).

7. Quan, L. et al. ACS Nano 8, 458-466 (2014).

8. Banciu, M., Metselaar, J. M., Schiffelers, R. M. \& Storm, G. J. Steroid Biochem. Mol. Biol. 111, 101-110 (2008).

9. Crielaard, B. J. et al. Int. J. Pharm. 416, 499-506 (2011).

10. Bartneck, M. et al. Biomaterials 37, 367-382 (2015).

11. Kroon, J. et al. Prostate 75, 815-824 (2015).

12. Gauthier, A. et al. Biomaterials 178, 481-495 (2018).

13. Deshantri, A. K. et al. J. Control. Release 296, 232-240 (2019).

14. A evaluation of the safety of Oncocort IV pegylated liposomal dexamethasone phosphate in patients with progressive multiple myeloma. ClinicalTrials.gov https://clinicaltrials.gov/ct2/show/ NCT03033316 (2017).

15. Zhang, J. et al. Front. Microbiol. 9, 915 (2018).

16. Metselaar, J. M., Wauben, M. H. M., Wagenaar-Hilbers,

J. P. A., Boerman, O. C. \& Storm, G. Arthritis Rheum. 48 2059-2066 (2003).

17. George, P. M., Wells, A. U. \& Jenkins, R. G. Lancet Respir. Med. https://doi.org/10.1016/S2213-2600(20)30225-3 (2020).

18. Kaier, K., Heister, T., Wolff, J. \& Wolkewitz, M. BMC Health Serv. Res. 20, 267 (2020).

19. Dasta, J., McLaughlin, T., Mody, S. \& Piech, C. Crit. Care Med. https://doi.org/10.1016/S2213-2600(20)30225-3 (2005).

\section{Acknowledgements}

T.L. and J.M.M. gratefully acknowledge support by the European Research Council (ERC) and the German Research Foundation (DFG). 